\title{
Mental Health and Substance Abuse Characteristics Among a Clinical Sample of Urban American Indian/Alaska Native Youths in a Large California Metropolitan Area: a Descriptive Study
}

\author{
Daniel L. Dickerson · Carrie L. Johnson
}

Received: 10 April 2010/Accepted: 17 November 2010/Published online: 16 December 2010

(C) The Author(s) 2010. This article is published with open access at Springerlink.com

\begin{abstract}
This study analyzes descriptive data among a clinical sample of American Indian/Alaska Native (AI/AN) youths receiving mental health services in a large California metropolitan area. Among 118 urban AI/AN youths, mood disorders $(41.5 \%)$ and adjustment disorder (35.4\%) were the most common mental health diagnoses. Alcohol $(69.2 \%)$ and marijuana $(50.0 \%)$ were the most commonly used substances. Witnessing domestic violence (84.2\%) and living with someone who had a substance abuse problem $(64.7 \%)$ were reported. The majority of patients demonstrated various behavior and emotional problems. Enhancing culturally relevant mental health and substance abuse treatment and prevention programs for urban $\mathrm{AI} / \mathrm{AN}$ youth is suggested.
\end{abstract}

Keywords Mental health · Substance abuse ·

American Indians · Native Americans · Trauma

\section{Introduction}

Psychiatric and substance use disorders among American Indian/Alaska Native (AI/AN) youths are significant problems affecting AI/AN communities (Beals et al. 1997; Dixon et al. 2007). For example, AI/ANs between the ages

D. L. Dickerson $(\bowtie)$

Integrated Substance Abuse Programs, University of California Los Angeles, 1640 S. Sepulveda Boulevard, Suite 200,

Los Angeles, CA 90025, USA

e-mail: daniel.dickerson@ucla.edu

D. L. Dickerson · C. L. Johnson

United American Indian Involvement, Inc.,

1125 W. 6th Street, Suite 103, Los Angeles, CA 90026, USA of 15 and 24 have the highest suicide rates in the United States compared to other racial/ethnic groups (CDC 2004). Although epidemiological studies are relatively limited with regard to AI/AN youth and specific psychiatric and substance use disorders, various studies conducted have identified concerning findings. For example, in a study conducted among indigenous adolescents from the northern Midwest and Canada, the lifetime conduct disorder rate was found to being more than twice the rates found in the general population (Whitbeck et al. 2008). Also, in a study analyzing data among a sample of Northern Plains adolescents aged 14-16 years and two comparison groups, AI youths demonstrated higher rates of disruptive behavior disorders (attention deficit-hyperactivity Disorder [ADHD], conduct disorder, and oppositional defiant disorder) than both comparison samples (Beals et al. 1997). These statistics are important since mental health problems experienced during childhood and adolescence may significantly impact growth and development, school performance, and peer and family relationships, as well as lead to increased suicide risk (Bhatia and Bhatia 2007).

Significant trends associated with alcohol and illicit drug use also have been identified among AI/AN youths (Dixon et al. 2007; Miller et al. 2008; Mitchell et al. 2008; Moncher et al. 1990). For example, AI/AN youths experience significantly higher rates of alcohol and illicit drug use, have an earlier onset of use, and experience more severe consequences of drug use compared to any other ethnic/ racial group in the United States (Dixon et al. 2007). Also, deaths attributable to alcoholism among AI adolescents and young adults, ages $15-24$, were found to be more than 15 times those of the same age group of all races combined (Mitchell et al. 2008). Furthermore, substance abuse among younger AI/AN children may be significant. For example, in a study comparing drug use between American Indian 
and non-Indian fourth- to sixth-grade students, American Indian children displayed patterns of drug use that paralleled those of older students (Miller et al. 2008). These trends are disquieting since drug and alcohol abuse by AI youths has been found to be associated with academic failure, delinquency, unemployment, and violent criminal behavior (Moncher et al. 1990).

During the past few decades, a notable shift in the AI/AN population has occurred, with nearly two-thirds of $\mathrm{AI} / \mathrm{AN}$ residing in urban areas (US Bureau of the Census 2000). An important historical explanation for this population shift is rooted in the Indian Relocation Act of 1956. This Act financed the relocation of individual American Indians and American Indian families to urban centers and provided funding to establish job training centers for them (Jaimes 1992). However, the relocation of AI/AN to urban areas has put them at risk for a variety of biopsychosocial problems (Evans-Campbell et al. 2006; Evans-Campbell and Walters, in press; LaFromboise et al. 1994). For example, the large numbers of AI/AN who moved to urban areas did not ultimately experience economic stability but rather found themselves homeless, unemployed, in poverty, and without a strong cultural base or community. The relocation of AI/AN to urban areas appears to have contributed to an inter-generational effect whereby successive generations of $\mathrm{AI} / \mathrm{AN}$ have demonstrated ongoing healthrelated disparities.

One of the most prominent risk factors for psychiatric and substance use disorders among AI/AN youths is exposure to traumatic events. Various studies have found that $\mathrm{AI} / \mathrm{ANs}$ are especially likely to experience a range of violent and traumatic events involving serious injury or threat of injury to self, or to witness such threats or injuries to others (Manson et al. 2005). For example, in a comparative literature review of youth having experienced at least one traumatic event (Boyd-Ball et al. 2006), 61\% Northern Plains youths described at least one traumatic event (Jones et al. 1997) compared with 16-47\% among other adolescent populations (Cuffe et al. 1998; Giaconia et al. 1995; Kilpatrick et al. 2000).

Traumatic exposure has also been a known risk factor for substance abuse among AI/AN youths. For example, childhood physical abuse has been shown to increase the odds of alcohol and drug dependence among a sample of Northern Plains Indians (Libby et al. 2004). Also, in a study by Gray (1998), psychological and physical trauma occurred in the everyday lives of youths in a sample of $\mathrm{AI} / \mathrm{AN}$ adolescents receiving substance abuse treatment (Gray 1998). A variety of psychosocial and environmental risk factors may increase substance abuse behavior among AI/AN youths. For instance, O'Connell et al. (2007) found substance use by parents, witnessing family violence, or having experienced other traumatic events before the age of 18 among AI/AN were associated with a higher level of drug and alcohol use severity than that found among AI/AN who were not exposed to these factors.

Issues associated with cultural identity and other psychosocial factors may influence mental health and substance abuse characteristics among urban AI/AN youth. For example, experiences of acculturative stress directly and indirectly related to the historically related trauma experienced by AI/ANs have been shown to result in poor mental health outcomes (Duran and Duran 1995). Furthermore, AI/AN may experience disruptions in their family relationships and may demonstrate an increase in their risk-taking behaviors which could threaten their health and well-being (Machamer and Gruber 1998). In addition, in a study conducted among urban AI adolescents, low self-worth was identified as a relevant factor in the genesis and maintenance of alcohol-related problems (Radin et al. 2006).

Thus, due to significant risk factors for psychiatric and substance use disorders among urban AI/AN youths, it is of increasing importance to conduct further research investigating psychiatric and substance abuse characteristics among urban AI/AN youths. The purpose of this descriptive study was to analyze substance abuse and psychosocial characteristics among a sample of AI/AN youths receiving mental health services at an urban clinic.

\section{Methods}

\section{Participants}

AI/AN youths receiving mental health services at an urban clinic in a large California city providing services specifically for AI/AN were included in this study. All participants $(n=118)$ were under 17 years of age when they began receiving mental health treatment services. However, due to reporting requirements of the national evaluation utilized in this study, a small number of individuals between the ages of 18 and 21 years were included in our analyses since the child and adolescent mental health treatment program continues to provide mental health treatment until patients are 21 years old. To be included in this study, AI/AN youth were required to have biological/psychosocial identity as an AI/AN based on both the subject's self-reported tribal identity and the judgment of the intake coordinator who interviewed participants. No information was obtained on blood quantum. To protect the confidentiality of these tribal members, we chose to not identify specific tribal groups (Norton and Manson 1996) or the clinic where data was retrieved. 


\section{Source of Data}

This study analyzes descriptive data among AI/AN urban youth who were receiving mental health care services from 2007-2008. Approximately 75\% of youth receiving services at this clinic are referred to mental health treatment from caregivers, parents, and family court. Services provided include individual and group counseling, participation in AI/AN cultural activities, and psychiatric treatment. The data were obtained from a series of assessments utilized by the National Evaluation of the Comprehensive Community Mental Health Services for Children and their Families Program, funded by the Child Adolescent and Family Services Branch, at the Substance Abuse and Mental Health Services Administration (SAMHSA 2010). This program utilizes the System of Care approach to services. This approach is a philosophy that utilizes "an approach to services that recognizes the importance of family, school and community, and seeks to promote the full potential of every child and youth by addressing their physical, emotional, intellectual, cultural and social needs." (SAMHSA n.d.). Approval for this study was granted by the UCLA, Institutional Review Board (IRB), South General Campus IRB (SGIRB): 00004474.

\section{Instruments}

Enrollment and Demographic Information Form (EDIF): The EDIF is an instrument that systematically collects descriptive information including demographic, diagnostic, and enrollment information on youth receiving mental health care services. Diagnostic and Statistical Manual of Mental Disorders (DSM)-IV criteria were utilized for psychiatric diagnostic information (American Psychiatric Association 1987). Axis I diagnoses were provided by a psychiatrist, psychologist, licensed clinical social worker, primary care physician, or other provider providing treatment to the client. Additional child diagnostic information was gathered using Diagnostic Classification of Mental Health and Developmental Disorders of Infancy and Early Childhood, Revised [DC:0-3 (DC:0-3R)] criteria (Zero to Three 2005).

Substance Use Survey-Revised (SUS-R): The SUS-R gathers information on history of having ever used alcohol, tobacco, and other drugs including cocaine (all forms), hallucinogens, marijuana, phencyclidine (PCP), ketamine (or Special K), 3,4-Methylenedioxymethamphetamine (MDMA/ecstasy), gamma-hydroxybutyric acid (GHB), inhalants, heroin, amphetamines/stimulants, narcotic pain medications, stimulant prescription medications, tranquilizers/benzodiazepines, barbiturates, alcohol, cigarettes, chewing tobacco/snuff, and over-the-counter medications. This survey includes frequency of use and age of onset. However, due to limited data on this sample regarding frequency of use and age of onset, these data was not included in the analyses.

Caregiver Information Questionnaire-Intake (CIQ-I): The CIQ-I gathers additional demographic information, as well as information on risk factors, family composition, custody status, service use history, and presenting problem(s) for children.

Child Behavioral Checklist (CBCL 6-18): The CBCL/ 6-18 obtains reports from parents, other close relatives, and/or guardians regarding the children's competencies and behavior/emotional problems (Achenbach and Rescorla 2001). The CBCL/6-18 has 118 items that describe specific behavior and emotional problems, plus two openended items for reporting additional problems. The CBCL/ 6-18 produces eight narrow-band syndrome scores: anxious/depressed, withdrawn/depressed, somatic complaints, social problems, thought problems, attention problems, rule-breaking behavior, and aggressive behavior; two broadband syndrome scores, internalizing and externalizing; and a total problem score. Parents provide information for 20 competence items covering their child's activities, social relations, and school performance and rate their child for how true each item was at the time the survey was taken and in the previous 6 months using the following scale: $0=$ not true (as far as you know), $1=$ somewhat or sometimes true $; 2=$ very true or often true.

\section{Results}

Participant Characteristics

A total of $118 \mathrm{AI} / \mathrm{AN}$ youth were included in this study. The gender composition in our sample was $57.6 \%$ male and $42.4 \%$ female. The average age was 9.6 years. Seventy-one $(60.1 \%)$ were $0-11$ years of age, $40(39.1 \%)$ were age $12-18$ years of age, and $6(0.9 \%)$ were $19-21$ years of age.

Prevalence of Psychiatric Disorders

With regard to DSM-IV Axis I psychiatric disorders, mood disorders $(41.5 \%)$ and adjustment disorder (35.4\%) were the most reported diagnoses in this sample, followed by posttraumatic stress disorder/acute stress disorder (23.1\%). Oppositional defiant behavior was reported in $10.1 \%$ of the sample, followed by attention-deficit/hyperactivity disorders $(4.6 \%)$, anxiety disorders $(4.6 \%)$, and learning, motor skills, and communication disorders $(3.1 \%)$.

Prevalence of Substance Use

Alcohol was the most commonly reported substance used by AI/AN urban youth (69.2\%), followed by marijuana 
(50.0\%), 3,4-Methylenedioxymethamphetamine (MDMA/ ecstasy) (15.4\%), amphetamines/stimulants (15.4\%), narcotic pain medications (15.4\%), cocaine (all forms; $15.4 \%$ ), cigarettes $(7.7 \%)$, hallucinogens $(7.7 \%)$, inhalants $(7.7 \%)$, stimulant prescription medications $(7.7 \%)$, and over-thecounter medications $(7.7 \%)$. No participants reported ever using chewing tobacco/snuff, PCP, ketamine, GHB, heroin, tranquilizers/benzodiazepines, or barbiturates.

\section{Psychosocial Characteristics}

Based on the CIQ-I, witnessing domestic violence was reported $(84.2 \%)$, followed by living with someone who had a substance abuse problem (64.7\%), living with someone who was depressed $(55.6 \%)$, living with someone who was convicted of a crime $(47.4 \%)$, living with someone who had a mental illness, other than depression $(29.4 \%)$, being physically abused $(26.3 \%)$, being sexually abused $(5.3 \%)$, and having run away $(5.3 \%)$.

Based on the CBCL 6-18, the majority of patients demonstrated behavior and emotional problems including rule-breaking behavior $(58.9 \%)$, feeling withdrawn $(59.1 \%)$, somatic complaints $(59.0 \%)$, feeling anxious/ depressed (57.8\%), attention problems (58.7\%), aggression problems $(62.2 \%)$, experiencing social problems $(57.5 \%)$, experiencing thought problems $(58.7 \%)$, internalizing problems $(57.3 \%)$, and externalizing problems $(59.8 \%)$.

\section{Discussion}

Results from this study conducted among a sample of urban AI/AN youth receiving mental health services revealed high rates of mood and adjustment disorders, alcohol and marijuana use, and traumatic exposure. Also, urban AI/AN youth in this study demonstrated various mental health symptoms including feeling withdrawn, aggressive problems, attention problems, and internalizing/ externalizing problems. Furthermore, many urban AI/AN youth in this study had witnessed domestic violence, lived with someone who had a substance abuse problem, lived with someone who was depressed, lived with someone who was convicted of a crime, and had histories of physical abuse.

Other studies of psychiatric and substance use characteristics among urban AI/AN adolescents are limited. However, in a study analyzing traumatic events and alcohol use disorders among AI adolescents and young adults, a higher likelihood of experiencing multiple severe traumatic events was found among those who reported that their parents used alcohol (Boyd-Ball et al. 2006). Also, traumatic exposure significantly increased their odds of alcohol use disorders for those who experienced $3+$ traumatic events compared to those with no traumatic events. Furthermore, the most frequently reported traumatic event type was witnessing trauma. Since witnessing domestic violence was also the most common traumatic event reported in our study among urban $\mathrm{AI} / \mathrm{AN}$ receiving mental health services, enhancing mental health treatment and substance abuse treatment and prevention programs targeting the effects of traumatic exposure, especially witnessing domestic violence, is suggested.

Urban AI/AN youth in this sample presented with various risk factors that may account for their high rates of mood and adjustment disorders. For example, based on these findings, traumatic exposure and residing with individuals who have significant mental health and substance abuse problems may result in elevated stress levels and elevated risk for abuse and/or neglect, resulting in mood and adjustment disorders. In addition, due to low socioeconomic status levels known to exist among AI/AN in large urban cities including Los Angeles (Ong and Houston 2003), accessing culturally relevant, affordable, and adequate mental health care may have been significantly challenging, resulting in more significant mental health problems. It is important to recognize the potential need for comprehensive mental health services for urban AI/AN youths with mental health problems. Thus, it is imperative that urban AI/AN youths have access to comprehensive culturally appropriate mental health services, including individual and family counseling, psychiatric treatment, community support groups, and culturally relevant treatment approaches, such as traditional healing services and activities.

PTSD and adjustment disorder rates were high among our sample of urban AI/AN youth. Potential risk factors may include high exposure to physical abuse and domestic violence. Also, high rates of parental substance abuse may be exacerbating these abuse issues, resulting in the experience of traumatic and abusive events by these youth. Thus, clinicians need to be aware of the potential for traumatic exposure among urban AI/AN children and adolescents who are presenting with significant mental health symptoms. Furthermore, clinicians serving this population need to be especially supportive in order to engage the patient in clinical work which could be especially challenging.

Exposure to traumatic events experienced by our sample of AI/AN adolescents warrants that effective culturally relevant treatments for PTSD, mood disorders, and adjustment disorders are provided. Potentially effective treatments include psychiatric medications, typically selective serotonin reuptake inhibitors (SSRIs). Among psychotherapies, one evidenced-based treatment for PTSD is Trauma-Focused Cognitive Behavioral Therapy 
(TF-CBT), an approach that provides, trauma-specific skills-based interventions for children and caregivers followed by more trauma-specific components with gradual exposure (Cohen et al. 2006). A cultural adaptation of TF-CBT for AI/ANs has been developed by Dee Big Foot and S. Schmidt, incorporating culturally relevant healing methods historically utilized in AI/AN communities (Bigfoot and Schmidt 2010).

High rates of mood and adjustment disorders among our sample of urban AI/AN and the known high risk for suicide among AI/AN youth, indicate the need for culturally relevant suicide prevention programs for AI/AN youth. Although many such programs have been well-received in AI/AN communities, limited studies have been conducted analyzing the effectiveness of specific suicide prevention programs for AI/AN youths. One program that has demonstrated effectiveness is the Zuni Life Skills Development curriculum (LaFromboise 1991). This curriculum is based on social cognitive theory and focuses on enhancing competency in a variety of life skills. The curriculum contains seven units: (1) Building self-esteem, (2) Identifying emotions and stress, (3) Increasing communication and problem-solving skills, (4) Recognizing and eliminating self-destructive behavior such as pessimistic thoughts or anger reactivity, (5) Receiving suicide information, (6) Receiving suicide intervention training, and (7) Setting personal and community goals.

The urban AI/AN youth receiving mental health services in our study demonstrated very high rates of alcohol and marijuana use. Reasons for these elevated rates may be related to their high exposure to psychosocial stressors identified in this study and the need to cope with these stressors. In addition, AI/AN youths residing in urban areas may experience unique issues associated with a shortage of family and community-based support networks, issues associated with their cultural identity within an urban environment that consists predominantly of non-AI/AN, and a shortage of comprehensive culturally appropriate prevention and treatment programs. Strengthening culturally grounded family and community supports, developing peer and mentoring programs for urban AI/AN youth, and addressing issues associated with cultural pride and identity may help reduce the initiation of drug and alcohol use among urban AI/AN youths. In addition, enhanced prevention and treatment programs targeting at-risk urban AI/AN youth experiencing these psychosocial stressors is urgently needed. In another study conducted among urban AI youths in the Southwest U.S., AI youths demonstrated steeper use trajectories in the amount and frequency of alcohol and marijuana compared with non-AIN youth (Dixon et al. 2007). Further studies analyzing the association of alcohol and marijuana use with various psychosocial stressors among urban AI/AN youth are needed.
Utilizing culturally relevant substance abuse prevention programs for urban AI/AN youth may assist towards decreasing the likelihood of alcohol and drug initiation. An approach designed as an after-school alcohol prevention program for urban youths, the Seventh Generation Program, is an urban after-school alcohol prevention program for youth in Colorado (Moran and Bussey 2006). The program blends general prevention approaches with culturally appropriate interventions including an emphasis on core AI/AN values and concepts associated with the Medicine Wheel of the Northern Plains tribes. Another relevant substance abuse prevention strategy for AI adolescents focuses on bicultural competence. Schinke and colleagues utilized concepts related to a bicultural approach indicating that AI youths need to acquire skills to be able to function in both AI/AN and non-AI/AN settings (Schinke et al. 1988). Incorporating cultural elements into prevention programs are important for AI/AN since developing cultural pride may enhance AI/AN youths' perceptions of their own value, thus motivating them to participate in healthy behaviors (Marsiglia et al. 2006; Vernon 2001).

Results from this study may be attributable to ongoing pervasive effects attributable to historically based trauma experienced by AI/ANs. According to the theory of historical trauma postulated by Maria Yellowhorse Brave Heart, various historically based political, social, and economic injustices experienced by AI/ANs throughout U.S. history may be contributing to ongoing problems associated with their high rates of mental health problems and substance abuse (Brave Heart 2005; Johnson 2006). Thus, a more comprehensive approach addressing mental health and substance abuse problems within a broader social framework is needed. For example, further focus on the need for additional economic opportunities, enhancing social and educational programs, and prioritizing AI/AN issues within the political, economic, and social framework can help decrease health-related disparities experienced by AI/ANs and the mental health and substance abuse problems among urban AI/AN youths. In addition, providing an opportunity for community discussion with regard to the histories and stories of local AI/AN urban individuals and families may assist in community cohesion, wellness, and healing. Further research is needed to address how the effects of historically based trauma may be presenting clinically with regard to the psychiatric and substance abuse problems among urban AI/AN youth.

\section{Limitations}

This study is subject to various limitations. First, it is restricted to one urban area within the United States and may not be representative of all AI/AN youths who are 
receiving mental health services in the U.S. Furthermore, self-reports of prior substance use were obtained rather than a diagnosis of abuse or dependence based on strict DSM criteria. Also, no comparison groups were included in this study, and statistical approaches analyzing the associations between mental health disorders and substance abuse with various risk factors were not utilized. The average age in our sample of urban AI/AN youths was young and may not capture the extent of mental health and substance abuse characteristics among AI/AN youths receiving mental health services. Also, to our knowledge, the cross-cultural applicability of the survey instruments used in this study has not been validated among AI/AN. In addition, further clinical information (e.g., treatment response rates) was not available in this study. However, we plan to conduct further analyses with regard to treatment response in subsequent studies, which should assist in furthering our understanding of issues raised in this preliminary study. Nonetheless, this study provides valuable information with regard to potential risk factors and mental health and substance abuse characteristics among urban AI/AN youths receiving mental health services, which can aid improvements in effective prevention and treatment approaches for this population.

In conclusion, results from this study demonstrate significant potential risk factors associated with mental health and substance abuse among urban AI/AN youths. Exposure to domestic violence and other stressors within the home environment should be addressed when treating urban AI/AN youth with mental health problems. Further development and delivery of culturally relevant prevention programs and comprehensive treatment strategies should also be considered. These programs and strategies should address historically rooted issues and ongoing health-related disparities experienced by this population. Further studies analyzing risk factors among urban AI/AN in other urban areas of the United States may enhance our understanding of mental health and substance abuse issues experienced by this population.

Open Access This article is distributed under the terms of the Creative Commons Attribution Noncommercial License which permits any noncommercial use, distribution, and reproduction in any medium, provided the original author(s) and source are credited.

\section{References}

Achenbach, T. M., \& Rescorla, L. A. (2001). Manual for the ASEBA school-age forms \& profiles. Burlington: University of Vermont, Research Center for Children, Youth, and Families.

American Psychiatric Association. (1987). Diagnostic and statistical manual of mental disorders, revised 3rd edn. Washington, DC: American Psychiatric Association.
Beals, J., Piasecki, J., Nelson, S., Jones, M., Keane, E., Dauphinais, P., et al. (1997). Psychiatric disorder among American Indian adolescents: prevalence in Northern Plains youth. Journal of the American Academy of Child and Adolescent Psychiatry, 36, $1252-1259$.

Bhatia, S. K., \& Bhatia, S. C. (2007). Childhood and adolescent depression. American Family Physician, 75, 73-80.

BigFoot, D. S., \& Schmidt, S. (2010). Honoring children-mending the circle: Cultural adaptation of trauma-focused cognitive-behavioral therapy children. Journal of Clinical Psychology, Special Issue: Culture-Sensitive Evidence-Based Practices, 66, 847-856.

Boyd-Ball, A. J., Manson, S. M., Noonan, C., \& Beals, J. (2006). Traumatic events and alcohol use disorder among American Indian adolescents and young adults. Journal of Trauma Stress, 19, 937-947.

Brave Heart, M. Y. H. (2005). Substance abuse, co-occurring mental health disorders, and the historical trauma response among American Indians/Alaska Natives. Research Monograph, Bureau of Indian Affairs, DASAP, Washington, DC.

Centers for Disease Control and Prevention, National Center for Injury Prevention and Control (producer). (2004). Web-based injury statistics query and reporting system (WISQARS) [Online]. Available online from: URL: http://www.cdc.gov/ncipc/wisqars/ default.htm, http://www.cdc.gov/ncipc/wisqars/default.htm.

Cohen, J. A., Mannarino, A. P., \& Deblinger, E. (2006). Treating trauma and traumatic grief in children and adolescents. New York: Guildford Press.

Cuffe, S. P., Addy, C. L., Garrison, C. Z., Waller, J. L., Jackson, K. L., McKeown, R. E., et al. (1998). Prevalence of PTSD in a community sample of older adolescents. Journal of the American Academy of Child and Adolescent Psychiatry, 37, 147-154.

Dixon, A. L., Yabiku, S. T., Okamoto, S. K., Tann, S. S., Marsiglia, F. F., Kulis, B., et al. (2007). The efficacy of a multicultural prevention intervention among urban American Indian youth in the southwest US. The Journal of Primary Prevention, 28, 547-568.

Duran, E., \& Duran, B. (1995). Native American post-colonial psychology (pp. 93-156). Albany: State University of New York.

Evans-Campbell, T., Lindhorst, T., Huang, B., \& Walters, K. L. (2006). Interpersonal violence in the lives of urban American Indian and Alaska Native women: implications for health, mental health, and helpseeking. American Journal of Public Health, 96(8), 1416-1422.

Evans-Campbell, T., \& Walters, K. L. (2010). Indigenist practice competencies in child welfare practice: a decolonization framework to address family violence and substance abuse among First Nations peoples. In Fong, R, McRoy, R., \& Ortiz Hendricks, C. (Eds.) Intersecting child welfare, substance abuse, and family violence: Culturally competent approaches. Alexandria, VA: CSWE Press; in press.

Giaconia, R. M., Reinherz, H. Z., Silverman, A. B., Pakiz, B., Frost, A. K., \& Cohen, E. (1995). Traumas and posttraumatic stress disorder in a community population of older adolescents. Journal of the American Academy of Child and Adolescent Psychiatry, 34, 1369-1380.

Gray, N. (1998). Addressing trauma in substance abuse treatment with American Indian adolescents. Journal of Substance Abuse Treatment, 15, 393-399.

Jaimes, M. A. (1992). The State of Native America: Genocide, colonization, and resistance. Cambridge, MA: South End Press.

Johnson, C. L. (2006). An innovative healing model: Empowering urban native Americans. In T. Witko (Ed.), Mental health care for urban Indians: Clinical insights from native practitioners (pp. 189-204). Washington, DC: American Psychological Association.

Jones, M. C., Dauphinais, P., Sack, W. H., \& Somerville, P. D. (1997). Trauma-related symptomatology among American Indian adolescents. Journal of Trauma Stress, 10, 163-173. 
Kilpatrick, D. G., Acierno, R., Saunders, B., Resnick, H. S., Best, C. L., \& Schnurr, P. P. (2000). Risk factors for adolescent substance abuse and dependence: Data from a national sample. Journal of Consulting and Clinical Psychology, 68, 19-30.

LaFromboise, T. D. (1991). Zuni life skills development curriculum. Palo Alto, CA: Stanford Health Promotion Resource Center.

LaFromboise, T. D., Berman, J. S., \& Sohi, B. K. (1994). American Indian women. In L. Comas-Diza \& B. Green (Eds.), Women of color: Integrating ethnic and gender identities in psychotherapy (pp. 30-71). New York, NY: Guilford Press.

Libby, A. M., Orton, H. D., Novins, D. K., Spicer, P., Buchwald, D., \& Beals, J. (2004). Childhood physical and sexual abuse and subsequent alcohol and drug use disorders in two AmericanIndian tribes. Journal of Studies on Alcohol, 65, 74-83.

Machamer, A. M., \& Gruber, E. (1998). Secondary school, family, and educational risk: Comparing American Indian adolescents and their peers. The Journal of Educational Research, 91, 357-369.

Manson, S. M., Beals, J., Klein, S. A., Croy, C. D., \& The AISUPERPFP Team. (2005). The social epidemiology of trauma among two American Indian reservation populations. American Journal of Public Health, 95, 851-859.

Marsiglia, F. F., Nieri, T., \& Stiffman, A. R. (2006). HIV/AIDS protective factors among urban American Indian youths. Journal of Health Care for the Poor and Underserved, 17, 745-758.

Miller, K. A., Beauvais, F., Burnside, M., \& Jumper-Thurman, P. (2008). A comparison of American Indian and non-Indian fourth to sixth graders' rates of drug use. Journal of Ethnicity in Substance Abuse, 7, 258-267.

Mitchell, C. M., Beals, J., \& Whitesell, N. R. (2008). Voices of indian teens team; pathways of choice team. Journal of Studies on Alcohol and Drugs, 69, 666-675.

Moncher, M. S., Holden, G. W., \& Trimble, J. E. (1990). Substance abuse among Native-American youth. Journal of Consulting and Clinical Psychology, 58, 408-415.

Moran, J. R., \& Bussey, M. (2006). Results of an alcohol prevention program with urban American Indian youth. Child \& Adolescent Social Work Journal, 24, 1-21.
Norton, I. M., \& Manson, S. M. (1996). Research in American Indian and Alaska Native communities: Navigating the cultural universe of values and process. Journal of Consulting and Clinical Psychology, 64, 856-860.

O’Connell, J. M., Novins, D. K., Beals, J., Whitesell, N., Libby, A. M., Orton, H. D., et al. (2007). Childhood characteristics associated with stage of substance use of American Indians: Family background, traumatic experiences, and childhood behaviors. Addictive Behaviors, 32, 3142-3152.

Ong, P. M., Sun, H.-G., \& Houston, D. (2003). The status of American Indian children in Los Angeles (November). Released by the Los Angeles American Indian Children's Council and the UCLA Ralph and Goldy Lewis Center for Regional Policy Studies.

Radin, S. M., Neighbors, C., Walker, P. S., Walker, R. D., Marlatt, G. A., \& Larimer, M. (2006). The changing influences of self-worth and peer deviance on drinking problems in urban American Indian adolescents. Psychology of Addictive Behaviors, 20, 161-170.

Schinke, S. P., Orlandi, M. A., Botvin, G. J., Gilchrist, L. D., Trimble, J. E., \& Locklear, V. S. (1988). Preventing substance abuse among American-Indian adolescents: A bicultural competence skills approach. Journal of Counseling Psychology, 35, 87-90.

Substance Abuse and Mental Health Services Administration. (2010). Systems of care. Available at http://www.systemsofcare.samhsa. gov/. Accessed April 3, 2010.

US Bureau of the Census. (2000). 2000 Census of the Population: General Population Characteristics-United States. Washington, DC: US Bureau of the Census; 2000.

Vernon, I. S. (2001). Killing us quietly: Native Americans and HIV/AIDS. Lincoln: University of Nebraska Press.

Whitbeck, L. B., Mansoo, Y., Johnson, K. D., Hoyt, D. R., \& Walls, M. L. (2008). Diagnostic prevalence rates from early to midadolescence among Indigenous adolescents: First results from a longitudinal study. Journal of the American Academy of Child and Adolescent Psychiatry, 47, 890-900.

Zero to Three, Diagnostic Classification of Mental Health and Developmental Disorders of Infancy and Early Childhood: Revised Edition (DC: 0-3 R), Washington, DC, 2005. 